\title{
A Case History of Broadband Processing of West of Shetland Data
}

R. Telling (Dolphin Geophysical), N. Riddalls (Dolphin Geophysical), A. Azmi (Dolphin Geophysical), S. Grion (Dolphin Geophysical) \& R.G. Williams* (Dolphin Geophysical)

\begin{abstract}
A number of approaches to increasing the bandwidth of seismic data have been adopted over recent years and have predominantly focussed on avoiding the reduction in signal strength and hence $\mathrm{S} / \mathrm{N}$ at the frequencies associated with the receiver ghost notch.. In particular, Williams\& Pollatos, 2011, showed test results indicating that a modern, hydrophone only streamer towed deep enough that it is well away from environmental noise, such as sea swell and boat generated noise, can have sufficient $\mathrm{S} / \mathrm{N}$ to be usable even within the receiver ghost notches. This observation relies on the sea surface not being a perfect mirror. Grion et al, 2013, showed for a 3D data set from the N. Sea that with a flat cable at $30 \mathrm{~m}$ the difference in data quality between a $4 \mathrm{~m}$ sea state and a flat calm was small and that both datasets had usable $\mathrm{S} / \mathrm{N}$ within the receiver ghost notch. This study again suggests that the sea surface does not behave as a perfect mirror.
\end{abstract}

Sablon et al, 2012, showed the impact of a variable depth streamer on multiple suppression and the modifications to de-multiple algorithms necessary. In this case, the recording geometry included a large change in streamer depth but a significant part of the streamer beyond the near offsets is very deep and gently sloping. In contrast, this paper demonstrates the effectiveness of conventional demultiple algorithms on a 2D survey from West of Shetland shot with a cable towed with a mild, linear slant of $1 \mathrm{~m}$ per $\mathrm{km}$ of offset from $20 \mathrm{~m}$ at the near offset to $30 \mathrm{~m}$ at the far offset. Attenuation of the multiples in this area is well known as challenging because of a hard sea bed with a variable depth and also complex geology including volcanics.

The results indicate that with this recording geometry - moderately deep and with only a mild gradient of cable depth - conventional de-multiple techniques can be used successfully while at the same time allowing broadband post-migration receiver de-ghosting with an overall processing turnaround time comparable to that for conventional processing projects. The complexity of the geology required a range of de-multiple techniques to be used including SRME, SWME (Shallow Water Multiple Elimination) and high resolution Radon.

\section{Acknowledgements}

We thank Dolphin Multi-client for permission to use these data.

\section{References}

Grion, S., Azmi, A., Pollatos, J., Riddals, N. and Williams, R.G. [2013] Broadband processing with calm and rough seas: observations from a North Sea survey. $83^{\text {rd }}$ Annual SEG Meeting Expanded Abstracts, 226-230.

Sablon, R., Russier, R., Hardouin, D., Gratacos, B., Soubaras, R. and Lin, D. [2012] Multiples attenuation for variable depth streamer data, shallow and deep water cases. EAGE Extended Abstracts, Y011.

Williams, R.G. and Pollatos, J. [2011] The key to increased marine seismic bandwidth. First Break, 30, 101-105. 